\title{
Eigenrecht und Relativität des Natürlichen bei Johannes Buridanus
}

\author{
Rolf Schönberger (München)
}

Daß die natürliche Wirklichkeit nicht wirklich, sondern nur Schein sei, dies versuchte Parmenides mit Argumenten zu erweisen, zu deren Prämissen es gehörte, daß das Undenkbare, das unmöglich zu Denkende nicht wirklich sein könne. Ohne hier das Problem erörtern zu wollen, ob denn diese "Theorie“ selbst konsistent, d. h. denkbar ist, sei damit nur daran erinnert, daß schon in der Thematisierung der „Natur“ keine Selbstverständlichkeit liegt. Der Naturbegriff der Griechen ist selbst nicht in seinem eigenen Sinn „natürlich“, da er als Vorkommnis nicht unter sich selbst fällt. Dies vermag auch der kulturgeschichtliche Vergleich zu zeigen: Wenn man die Texte des Judentums betrachtet, dann muß man konstatieren, daß dort ein semitisches Äquivalent für den Begriff „Natur“ überhaupt fehlt. Er fehlt sogar in der sog. Sapientialliteratur, obwohl diese den Hellenismus unbestrittenermaßen sonst vielfältig voraussetzt. Wenn man etwa liest, wie Jesus Sirach (Kap. 42/43) die Herrlichkeit der Sonne, des Mondes, der Sterne und einer Reihe anderer Erscheinungen der - „Natur" möchte man sagen -, preist, ist es wahrhaft erstaunlich, daß er einen entsprechenden Terminus gleichwohl nicht verwendet. Der antike Begriff „Natur“ bezeichnet seit der Antike eine wesentliche Form von Wirklichkeit und einschlußweise ebenso eine fundamentale Einstellung zur Welt überhaupt. Das Ganze der Wirklichkeit wird aber nicht nur auch in der Antike, sondern hier zum ersten Mal als physis bzw. natura begriffen. Der Charakter eines von sich aus nicht selbstverständlichen Unternehmens ergibt sich nach diesen ganz knappen Hinweisen also zum einen aus seiner geschichtlichen Partikularität und zum anderen aus der griechischen Radikalität des philosophischen Fragens, in welcher der Sinn der Rede von Natur zumindest in einer Phase kritisiert wurde, in der aber durchgängig die angemessene Thematisierung kontrovers blieb.

Im großangelegten Unternehmen seiner Naturphilosophie hat Aristoteles jene Prämisse des Eleatismus von der Unwirklichkeit des Undenkbaren zwar konzediert, jedoch zu zeigen versucht, daß physis, soll heißen die physei onta wirklich denkbar sind. Man kann sie genauerhin dann denken, wenn Bewegung als der auszeichnende Grundzug des Natürlichen verständlich zu machen ist. Aristoteles gelingt dies, indem er auf eine 
Differenz im Begriff des Seins aufmerksam wird. Es gibt nicht bloß eine Weise, wie etwas etwas ist: Es kann beispielsweise etwas auch der Möglichkeit nach etwas sein oder tun. Mit Hilfe dieser Unterscheidung läßt sich Bewegung als eine antizipatorische Struktur bestimmen, so daß man - ohne die Subtilität und argumentative Kraft der aristotelischen Überlegungen hier analysieren zu können - zumindest versucht ist, sich die paradoxe Formulierung zu erlauben: Die Möglichkeit, Natur zu denken, beruht auf der Möglichkeit, Möglichkeit zu denken.

Damit ist allerdings weder der logische Status noch der Gehalt des Naturbegriffs geklärt. In der Regel werden Begriffe bestimmt, indem sie gegen andere abgegrenzt, eben definiert werden. So kann auch das, was das natürlicherweise Seiende auszeichnet, nur bestimmt werden, wenn man es gegen anderes abhebt. Indem aber mit der Rede „von Natur“ zugleich etwas gemeint ist, was durch Natur bestimmt ist, enthält der Begriff der Natur bereits selbst eine Beziehung auf anderes. Buridan sagt es im übrigen völlig klar, daß „Natur“ ein Relationsbegriff ist. ${ }^{1}$ Solche Gegenbegriffe sind für Aristoteles wie für die Griechen überhaupt techne, nomos, praxis etc. Von dieser Art ist der Begriff Natur meistens - nicht immer geblieben: nämlich bestimmt zu sein durch seine wechselnden Gegenbegriffe: Natur - Kunst; Natur - Wille; Natur - Geist; Natur - Freiheit; Natur - Gnade, d. h. „Über“-natur. Die Eigentümlichkeit des Naturbegriffs besteht jedoch darin, daß er gerade nicht durch exklusive Bestimmungen gegen diese anderen eingegrenzt wird, sondern sie in der Regel in gewisser Weise mit umschließt. Wenn daher in der Thematisierung einer solchermaßen verstandenen Natur ein universeller Anspruch impliziert sein sollte, so ist die Konfliktmöglichkeit mit einem an der biblischen Schöpfungslehre orientierten Denken bereits abzusehen. Die folgende Zuwendung zu Johannes Buridan soll jedoch nicht primär unter diesem globalen Aspekt stehen, da sein Denken selbst bereits eine Reaktion auf diesen Konflikt und dessen gedankliche und institutionelle Austragung darstellt. Zunächst muß vielmehr der begriffliche Gehalt und der logische Status des Begriffes „Natur“ und „natürlich“ im Vordergrund stehen. Dabei wird sich allerdings zeigen, daß der Status der buridanischen $\mathrm{Na}$ turbetrachtung sich nicht interpretieren läßt, ohne jenen, die sog. Hochscholastik tiefgreifend bestimmenden, Konflikt als Folie zu benutzen.

Es ist zunächst wichtig zu sehen, daß der oben kurz skizzierte und für Aristoteles grundlegende Status des Naturbegriffs ${ }^{2}$ auch noch für Buridan

\footnotetext{
1 Phys. II, 4 (31 va; vb sq.); Met. VII, 1 (42rb): „hoc nomen "natura" quamvis supponat pro substantia, tamen non est absolutum, sed dicitur relative ad motum; hoc patet II Physicorum quia diffinitur per motum, ut quia natura est principium movendi illud in quo est primum per se et non per accidens." [Bei den Schriften Buridans werden die üblichen Ausgaben (bzw. deren Nachdrucke) und Siglen verwendet.]

2 Cf. R. Spaemann, Natur, in: Philosophische Essays, Stuttgart 1983, 19-22.
} 
zentral bleibt. Die ausführlichste und am stärksten differenzierende Bedeutungsanalyse findet sich seinem Metaphysik-Kommentar anläßlich der Frage, ob wir über die Prinzipien allen Wissens „natürlicherweise“ verfügen. Was es heißt, auf eben diese Weise etwas zu haben, bestimmt Buridan durch sieben Gegenbegriffe: "Natürlich" ist 1. das, was nicht zufällig ist, sondern aus der intentio agentis hervorgeht; 2. das, was nicht gewaltsam, sondern aus einer natürlichen (soll wohl heißen inneren) Neigung hervorgeht; 3. das, was nicht künstlich ist; 4. das, was nicht übernatürlich (supernaturale seu miraculose) ist; 5. das, was nicht willentlich ist; 6. das, was nicht ungemäß (disconveniens) ist, wie etwa die Wärme dem Wasser; 7. das, was nicht erworben ist, sondern ursprünglich zugehört. ${ }^{3}$ Auch in seinem Politik-Kommentar hebt Buridan die innere Differenz im Naturbegriff häufiger hervor. ${ }^{4}$ Schon die dritte Frage im ersten Buch widmet Buridan einem in dieser Weise gestellten Problem: utrum civitas sit a natura? Wie Buridans Beantwortung der Frage zeigt, kann man sich nicht damit begnügen, das, was ,von Natur" ist, gegen ein anderes abzugrenzen, sei es Konvention, Wille oder Gewalt. Das „,von Natur“ hat nämlich auch in sich bereits mehrere Bedeutungen; die Präposition „a“ legt Buridan dabei zunächst auf einen kausalen Sinn fest.

Natur als „eine Weise, etwas zu sein“ zu begreifen schließt ein, daß dadurch ein Ding gerade nicht klassifiziert wird, sondern andere, davon unterschiedene Momente an diesem zugelassen werden. Dies ist zwar einerseits bei Aristoteles noch offenkundig, wie W. Wieland zu Recht hervorgehoben hat, ${ }^{5}$ doch beruht es andererseits auf der Voraussetzung, von Weisen des Seins zu reden - eine Präsupposition, die im Nominalismus wohl nicht widerlegt, jedoch obsolet geworden ist. ${ }^{6}$ Auch hier bedarf es keiner Interpretationssophistik, sondern des schieren Verweises auf

\footnotetext{
3 Met. II, 2 (9vb-10ra); ähnlich Eth. II, 2 (23ra): „naturale aliquando distinguitur con/ra casuale, aliquando contra violentum, aliquando contra accidentale, aliquando contra supernalurale, aliquando contra disconveniens, aliquando contra animale seu voluntarium" - worauf VIII, 18 (184va) nochmals verwiesen wird.

4 Pol. I, 3 (4ra); II, 1 (25ra-rb).

5 Wieland unterstreicht in seinen Untersuchungen (Die aristotelische Physik, Göttingen 1962), daß der aristotelische Begriff der Natur als dasjenige, was das Prinzip seiner Bewegung in sich hat, nicht als reine Selbstbewegung mißverstanden werden darf. Das von Natur Seiende hat nur einen Bewegungsursprung in sich: p. 234; cf. p. 236; 245; 260 .

"Ein zugleich philosophischer wie historischer Zugang zu vergangenen Problemdiskussionen hat immer mit beiden Formen des In-den-Hintergrund-tretens zu rechnen, auch wenn aus der Eigenperspektive der Scholastik dieser Prozeß als ein bloß faktischer sozusagen nicht vorgesehen ist, und auch wenn ein weitgehend herrschend gewordener Habitus der Auslegung die Reflexion auf das Verhältnis von Kritik und Kritisiertem aus der Perspektive des letzteren allzu oft übergeht; cf. vom Vf., Realität und Differen\%. Ockhams Kritik an der distinctio formalis, in: Die Gegenwart Ockhams, Weinheim 199(), ed. IX. Vossenkuhl-R. Schönberger, 97-122; bes. p. 109 sqq.
} 
Buridan-Texte, um zu zeigen, daß Buridan dies immer noch sagen kann. Diejenige Disponierung zum sittlich gelungenen Handeln, für welche die deutsche Sprache mittlerweile nur mehr ein altmodisch gewordenes Wort, nämlich Tugend, zur Verfügung hat, wird aristotelisch gerade so gedacht, daß es weder durch Natur ausschließlich noch ohne Natur definiert werden könnte. Daher bleibt Buridan auch hier durchaus im aristotelischen Rahmen, wenn er sagt: „virtutes fiunt in nobis per naturam inchoative ... per doctrinam excitative sive provocative ... per consuetudinem perfective." 7 "Natürlich" kann daher etwas bereits in einem anfangshaften wie auch in einem vollendeten Sinne sein. ${ }^{8}$

Aus dem bisherigen läßt sich also zunächst folgendes festhalten: Natur wird nicht primär begriffen als eine bestimmte Art oder Sphäre oder Region von Wirklichkeit, sondern als eine Weise von Wirklichkeit. Nicht um bestimmte Phänomene überhaupt zu klassifizieren, sondern um bereits sprachlich fixierte Phänomene in ihrem Status zu bestimmen, verwendet man in diesem Kontext die Rede von Natur. Auf dieser elementaren Ebene ist Buridans Naturbegriff seinem innerem Gerüst nach also noch der aristotelische.

Wie allerdings erst aus anderen (und wohl späteren) Texten hervorgeht, behält Buridan nicht einfach auch inhaltlich die aristotelischen Komplementärbegriffe bei. Zu nennen ist hier erstens die Einschmelzung der Unterscheidung von Natur und Kunst ${ }^{9}$ und zweitens die Antithese von Natur und Freiheit. Obwohl Buridan nicht müde wird zu erklären, daß der Wille „von Natur“ auf das Gute ausgerichtet ist ${ }^{10}$ so wie der Intellekt auf die Wahrheit, ${ }^{11}$ hält er doch den Unterschied fest, daß der Wille gar nichts Unbekanntes, also nicht durch den Intellekt Vermitteltes zu wollen vermag, während umgekehrt der Intellekt sich zwar irren kann, für Buridan jedoch in dessen finaler Austichtung auf Wahrheit doch so etwas wie eine Verbürgung seiner Wahrheitsfähigkeit liegt. Die Alternativität von Natur und Wille mag durch Duns Scotus zusätzliche Prominenz gewonnen haben, doch war die Diskussion um die göttliche Weise des Hervorbringens so

Fith. II, 1 (22ra).

${ }^{8}$ Pol. IV, 17 (61va): „Et boc dicit Philosophus II Ethicorum ubi dicit, quod virtus inest a natura bominibus iniciative"; I, 3 (4ra).

" Phys. II, 1.

1" Ebenso ist er „von Natur“ frei: Quaestiones super de caelo et mundo II, 5 (ed. Moody p. 147): ,voluntas enim naturaliter libera est, et potest se libere determinare ad quam partem voluerit, sine necessitate alterius causae determinantis."

1 Fth. I, 11 (11va): "veritas secundum quod de ea loquimur bic est conformitas nostrae cognitionis aut etiam nostri sermonis ad rem et baec veritas est perfectio ac bonum nostri intellectus." Phys. I, 4 (6va); ib., f. 5va; Met. I, 5 (5vb); Phys. I, 4 (6va); I, 15 (19ra); Eth. VI, 1 (116va); Periherm. II, 11 (ed. Van der Lecq p. 101). Angesichts dieser ziemlich drastischen Formulierungen hat A. Maier sogar von einer Art „Wahrheitsinstinkt“ gesprochen: Das Problem der Evidenz, in: Ausgehendes Mittelalter, Rom 1967 II p. 394 n. 52. 
allgemein, daß hier ein spezifisch scotischer Einfluß die Größenordnung der Verschiebung verkennen würde. ${ }^{12}$

Eine der genannten Arten von komplementären Entgegensetzungen spielt allerdings nur im vermutlich frühen Politik-Kommentar eine gewisse Rolle, späterhin jedoch überhaupt keine mehr. Selbst die dabei zu verwendende Benennung ist nicht die buridanische, sondern notgedrungen eine viel später geprägte: Natur und Geschichte. Der Aspekt des Geschichtlichen, genauer des im forcierten Sinne verstandenen Heilsgeschichtlichen fungierte immer wieder als kritischer Begriff, um die Selbstverständlichkeit der Rede von der Natur zu unterlaufen. Gemeint ist hier insbesondere jener Einwand, bestimmte Phänomene im Zusammenhang des Menschen (etwa auch seine Art zu erkennen) ließen sich nicht völlig unbesehen als „natürlich“ unterstellen; wenn Aristoteles dies in Unkenntnis der Geschichte von Urstand und Fall des Menschen tue, so sei dies einer der Sachverhalte, wo gegenüber einer aristotelisierenden, aber christlichen Theologie ein prinzipieller Vorbehalt bleiben müsse. ${ }^{13}$ Vor diesem Hintergrund könnte folgender kurzer Hinweis Interesse verdienen: In der Frage, ob irgendein Mensch von Natur ein Sklave sei (Pol. I, 6), nimmt Buridan ganz selbstverständlich im Interesse einer Konkordanz von christlichen Lehrern und Aristoteles die Rede von einem ursprünglichen status innocentiae in Anspruch. ${ }^{14}$ Auch wenn Buridan dort mehrfach die Unterscheidung von gefallener und nicht gefallener Natur verwendet, so zieht er daraus doch gleichwohl keine grundsätzlichen Folgerungen. Man vermag jedoch die Selbstverständlichkeit dieser Inanspruchnahme nur dann angemessen einzuschätzen, wenn man im Auge behält, daß das Argument von der möglichen Geschichtlichkeit des scheinbar von Natur so Seienden zu den wichtigsten Einschränkungen der philosophischen Vernunft gehörte. Es liegt also auch hier keine Reaktion auf Duns Scotus vor, denn spätestens dieser hatte diese Selbstverständlichkeit problematisch gemacht. Die cxtreme Gegenposition zu Scotus nimmt in dieser Hinsicht wohl Meister

${ }^{12} \mathrm{G}$. Krieger hat mehrfach scotische Ansätze als Hintergrund für Diskussionen Buridans herangezogen: G. Krieger, Der Begriff der praktischen Vernunft nach Johannes Buridanus, Münster 1986, 11 sq.; 13 sq. Die Stellung und Bedeutung der philosophischen Fthik bei Johannes Buridanus, in: Medioevo 12 (1986) 131-195, bes. p. 137-141; 154; 174; 176. Es scheint mir jedoch noch klärungsbedürftig, inwieweit es sich dabei nicht bloß um eine interpretatorische Kontrastierung, sondern um eine reale, historisch festzumachende scotische Beherrschung der Diskussionslage an der Pariser Artes-Fakultät des 14. Jahrhunderts handelt - so wie sich etwa für Buridan die Formen der Berücksichtigung etwa des Thomas oder des Albertus Magnus angeben ließen.

13 C.f. Et. Gilson, Jean Duns Scot, Paris 1952, 11 sqq.; 61-62; 67 sqq.; 521 sqq.; cf. im Index s. v. „état".

$1+$ Pol. I, 6 (8ra-8vb); cf. I, 7 (10ra; 10va); VIll, 5 (114rb). 
Eckhart ein, in dessen beiden Genesis-Kommentaren man vergebens nach einer Auslegung der Geschichte vom Sündenfall sucht. ${ }^{15}$

Die für die Bestimmung von Buridans Naturphilosophie bei weitem wichtigste Antithese ist jedoch zweifellos diejenige von naturale und supernaturale ( $=$ miraculose). ${ }^{16}$ Entscheidend ist hier allerdings nicht, daß diese bei Aristoteles nicht vorkommt, sondern daß Buridan sie verwendet, um eine Naturphilosophie begründen zu können, die unter den spezifischen Bedingungen des 14. Jahrhunderts zu bestehen vermag. Was es nämlich heißt, die "Natur" auf natürliche Weise zu betrachten, kann jetzt nicht mehr wie bei Aristoteles selbst nur gegen eine alternative Naturspekulation (wie etwa den platonischen Timaios oder den vorsokratischen „Materialismus") definiert werden, vielmehr muß die Betrachtungsweise, welche unter den neuen Rücksichten jetzt „natürlich“ heißen kann, gegen einen hypertrophen Theologismus verteidigt werden. Um dies nur mit jeweils einem Stichwort in Erinnerung zu rufen: Ein Ansatz wie der des Bonaventura setzt alle Aussagen unter den Maßstab letztgültiger Approbierbarkeit. Etwas ist entweder wahr und dann schlechthin akzeptabel, oder aber falsch, d. h. gefährlich. Selbst Aegidius Romanus hat seine Skepsis gegenüber dem Versuch zum Ausdruck gebracht, das aristotelische Denken auf einen partikularen Anspruch zu beschränken, aber unter der Voraussetzung einer solchen Einhegung intakt zu lassen. ${ }^{17}$ Bonaventura hat jenen Anspruch u. a. insbesondere im Zusammenhang einer symbolistischen Auslegung des Hexaëmeron (!) formuliert. Nur ein Seitenblick sei darauf geworfen, daß seit dem 12. Jahrhundert immer wieder naturphilosophische Durchdringungen des Sechstagewerkes versucht wurden - wobei immer noch zu fragen bliebe, was im Zusammenhang eines völlig uniken Geschehens der Terminus Natur besagen könnte. Der vielleicht letzte großangelegte Versuch, Naturphilosophie und spekulative Exegese zur Konkordanz zu bringen, liegt wohl im Werk des Meister Eckhart vor, insbesondere in seinem Kommentar zum Johannesevangelium. ${ }^{18}$ Weder eine unter theologischen Maßstäben zutagetretende und dann aus einer be-

15 Hingegen bestimmt Eckhart bereits die Vielheit als solche als einen „Fall“ (casus) vom Einen und durch dessen Konvertibilität mit den anderen Transzendentalien demzufolge auch als einen Fall vom Wahren, Guten und dem Sein: Joh. n. 526 (LW III p. 456 sq.); n. 692 (p. 609); n. 713 (p. 623).

16 Vgl. M. H. Reina, L'ipotesi del ,casus supernaturaliter possibilis' in Giovanni Buridano, in: La filosofia della natura nel medioevo, Milano 1966, 683-690; G. Federici Vescovini, La concezione della natura di Giovanni Buridano, ib., p. 616-624.

17 De erroribus philosophorum I, 9 (ed. J. Koch p. 8): „nec valeret, si aliqui vellent excusare ipsum, quia loquitur per viam naturae, cum crediderit nibil novi posse immediate a Deo procedere, sed omne novum contingere per viam motus et per operationem naturae."

18 Eckhart, Joh. n. 3 (IW III p. 4); cf. n. 6 (p. 8); n. 13 (p. 12); n. 125! (p. 108); n. 137 (p. 116); n. 142 (119 sq.); n. 173 (p. 142); n. 185 (III p. 154 sq.); n. 275 (III p. 231); n. 492 (p. 424); n. 510 (p. 441); n. 668 (p. 580 sq.); eine Erläuterung dieses Programms bei L. Hödl, Naturphilosophie und Heilsbotschaft in Meister Eckharts Auslegung des JohannesFivangeliums, in: La filosofia della natura nel medioevo, Milano 1966, p. 641-651. 
stimmten Prämisse zu verwerfende Insuffizienz, noch eine von theologischen Interessen geleitete Konkordanz können selbstverständlich die Lcitideen eines Magisters der Philosophie im 14. Jahrhundert sein. Es wäre jedoch ganz falsch, diese neue Situation nur von dem Gegensatz von Theologie und Philosophie her zu interpretieren. Dies würde dem begrifflichen Status des neuen Gegensatzes natürlich - übernatürlich nicht gerecht:

Wenn es nämlich richtig ist, daß sich die bisher betrachteten strukturellen Momente des Naturbegriffs noch im Horizont des aristotelischen Konzeptes bewegen, so sind doch gleichwohl damit nicht alle relevanten Bestimmungen zusammengetragen. „Natürlich" ist nämlich, wie bereits angedeutet, für Buridan auch eine Betrachtungsweise, man könnte sagen: eine Methode. Diese ist nun jedoch gerade nicht mehr im Sinne der schon genannten Bedeutungen „natürlich“, sondern wird auf der Basis einer bewußten Entscheidung angewandt. Zwar hatte auch die Antike schon von einer natürlichen Theologie gesprochen; damit war jedoch eine Art der Betrachtung gemeint, die ungeachtet partikulärer Gesichtspunkte die Natur der Dinge selbst thematisiert und daher von allgemeiner Gültigkeit sein kann. Nun hat sich erstaunlicher Weise das Christentum fast ausschließlich mit dieser Form der "Theologie“ auseinandergesetzt, aber erst seither spricht man von ,natürlicher" Vernunft. Diese wiederum meint eine bestimmte Form der Wahrheitsverbürgung, welche definiert ist durch den Ursprung bzw. die Weise des Zustandekommens von Wahrheit. Wenn man nämlich jenen Ausdruck paraphrasiert als "mit den Mitteln der Vernunft", dann ist hiermit an einen Gegensatz gedacht, für den sich mit den sogenannten Mitteln der Vernunft gar keine Kategorie finden läßt, nämlich jeweils einzelne Handlungen Gottes, sei es eine Offenbarung, seien es außergewöhnliche Ereignisse, die auf einen Ursprung außerhalb der Natur und diesen darin zugleich als der Natur schöpferisch überlegen zeigen. Wo immer sich im Mittelalter eine selbständige Philosophie als Manifestation der natürlichen Vernunft etabliert, tut sie dies mit einer Kategorie, welche einen durch diese Selbständigkeit nicht definicrbaren Ursprung hat. Es fällt daher auch nicht schwer, für diese Einstellung Belege aus der Theologiegeschichte anzuführen. ${ }^{19}$ Am bekanntesten ist jedoch wohl jene Passage aus Albertus Magnus, die Buridan sogar selbst einmal anführt: „Nam sicut dicit Albertus primo de generatione: quid ad nos de dei miraculis, cum de natura loquimur. " ${ }^{20}$ Auf diesen Text haben sich ansonsten insbesondere averroistische Autoren berufen. ${ }^{21}$

19 Thomas von Aquin, sth. I, 76, 5 ad 1: ,in constitutione rerum naturalium non consideratur quid Deus facere possit, sed quid naturae rerum convenial"; mit Berufung auf Augustinus, De gen. ad litt. II, 1, 2; PI. 34, 263.

21" Eth. I, 17 (17rb): Albertus, de gen. I, 22 (IV, 363 Borgnet); ähnlich Fth. I, 7, 5 ( ( II, $114 \mathrm{~b}$ Borgnet).

2i Siger von Brabant, de an. intell. 3 (Bazán 83 sq.); Johannes von Jandun, in Met. I, 16 (13ra); cf. B. Nardi, La posizione di Alberto Magno di fronte all'averroismo, in: Riv. crit. di storia della filos. 2 (1947) 197. 
Auf die Frage, was denn Natürlichkeit ihrer Form nach sei, haben wir oben sehr allgemein gesagt, sie ist eine Weise. Sie ist zwar auch eine Region, aber primär eine Weise zu sein, und eine Region nur, insofern bestimmtes Seiendes durch diese Weise zu sein primär bestimmt ist. Dic oben genannten Entgegensetzungen sind keine epochalen Abgrenzungen, sondern sind gleichzeitige. Als Weise der Betrachtung, als Methode mithin, wird nun Natur zu derjenigen Denkform, die sich bei Buridan in markanter Weise vertreten findet. Eine solchermaßen ansetzende Untersuchung nimmt Natur als eine bestimmte Möglichkeit. Die Natur bestimmt darin einen Modus von Möglichkeit, der abzugrenzen ist gegen einen anderen Modus. Dieser ist terminologisch als eine übernatürliche, nur in der Form des Wunders realisierbare Möglichkeit gefaßt, der Sache nach jedoch als die eigentliche, jedenfalls aber doch als die umfassende Möglichkeit bestimmt. Die göttliche Allmacht ist faßbar als zweiter Aspekt, der jedoch mit diesem äquivalent ist. Sie ist der Intension nach gefaßt als Denkmöglichkeit und betrifft daher den weitesten Raum von Möglichkeit. Sie ist aber andererseits nicht reine Gedachtheit oder gar von ontologisch weiter nicht ausweisbarem Status. Vielmehr ist sie ein reales Vermögen, das als reale causa zu fungieren imstande ist. ${ }^{22}$ Man muß die Frage stellen, was dies eigentlich naturphilosophisch erbringt. Ergeben sich daraus auch inhaltliche Konsequenzen oder handelt es sich nur um ein bestimmtes theologisches Vorzeichen vor der Klammer der Naturphilosophie, durch welches die internen Verhältnisse zwar noch einmal von außen bestimmt sind, aber für die Binnenstruktur ohne Implikationen bleibt?

Welche Funktion hat also der Allmachtsgedanke bei Buridan? Er wird bei ihm zum negativen Kriterium des Natürlichen. Der Gedanke einer Ursache, die jederzeit in jeden Prozeß einzugreifen imstande ist, aber auch als imstande gedacht wird, eine andere als die vorfindliche Welt zu etablieren, schafft einen ungeheuren Freiraum zur Ausbildung von Hypothesen. Die Kosmologie bedarf, um den Status ihrer Aussagen zu bestimmen, hypothetischer, im Modus des Potentialis formulierter Erwägungen. Erst wenn die naturphilosophische Theoriebildung diese Phase der Hypothesenbildung durchlaufen hat, kann entschieden werden, ob eine Behauptung einen faktischen oder einen notwendigen Charakter hat. Der Modalitätsstatus wird durch die hypothetisch möglichen oder nicht möglichen Varianten sichtbar.

Das Verfahren methodischer Separation bestimmt Buridans Philosophie zwar im Ganzen, doch nicht überall in derselben Weise. Das zunehmende

22 Phys. I, 15 (19rb): „agens non dicitur omnipotens ex eo quod possit facere impossibilia fieri vel eliam ex eo quod possit possibilia fieri aliter quam sint possibilia fieri: Deus non potest facere alium Deum sibi aequalem. Sed ipse est omnipotens quia potest facere omnia possibilia fieri ex modo quo sunt possibilia fieri; immo omne quod fit ipse facit et omne quod fiet ipse faciet; sed boc erit ex subiectis praesuppositis et concurrentibus aliis agentibus particularibus"; I, 22 (26ra). 
Kontingenzbewußtsein, welches in der nominalistischen Ethik zu grotesken Erwägungen über die mögliche Geltung der Vorschrift des Gotteshasses geführt hat, wird in dem monumentalen (aber gleichwohl fragmentarisch gebliebenen) Ethik-Kommentar überhaupt nicht explosiv. Dies gilt nicht gleichermaßen für die naturphilosophischen Abhandlungen. Erst durch die Ausklammerung des über-natürlicherweise Möglichen konstituiert sich eine Philosophie von der Natur. Eine solche methodische Separation bezieht sich trotzdem notwendig, wenn auch negativ, auf das Ausgeklammerte. Dies gilt zunächst mit Bezug auf einzelne aristotelische Notwendigkeitsbehauptungen. Für einen Lehrer der artes im 14. Jahrhundert wird es unausweichlich, den Sinn des dabei unterstellten Notwendigkeitsbegriffs zu klären. Buridan kann jetzt sagen, die behaupteten Notwendigkeiten würden zwar gelten, doch nur natürlicherweise. Mit Bezug auf den Ursprung eben dieser Natur wird es unmöglich, diese Art von Notwendigkeit mit dem Notwendigen im absoluten Sinne zu identifizieren.

Es scheint allerdings, daß die Reflexion auf den Status von Aussagen nicht von ungefähr gerade in der Naturphilosophie eine solche überragende Rolle spielt. Nicht zufällig deshalb, weil sich die Naturphilosophie des Aristoteles nicht allein inhaltlich und methodisch von der platonischen tiefgreifend unterscheidet, sondern auch durch ihren Anspruch. Platon hatte sich durch das Problem der Möglichkeitsbedingungen von Wissen, von „Sich-Verstehen-auf“ genötigt gesehen, von Ideen zu reden; wenn jedoch diese deshalb tauglich sind, diese Bedingungen zu erfüllen, weil sie selbst in gewisser Hinsicht dessen Gegenstände bilden, dann kann das Bewegte zwar - wie der Phaidon vorführt - durch seinen finalen Bezug auf jene Idee indirekt und je für sich erfaßt, jedoch nicht als kosmologisches Ganzes zum Gegenstand des Wissens im strikten Sinne werden. Platon spricht denn auch konsequenter Weise von eikotes mythoi. ${ }^{23}$ Aristoteles hingegen erhebt in De caelo den dort oftmals unterstrichenen Anspruch rigoroser, d. h. absoluter Notwendigkeit. ${ }^{24}$ Dies konnte in der christlichen Tradition nicht ohne tiefgreifende Korrektur rezipiert werden. Zwar ist es wohl überflüssig zu sagen, daß in der aristotelisch verstandenen Natur es sogar ein Prinzip der Unbestimmtheit und im Gedanken der Interferenz diverser Faktoren sich auch ein Raum für das Zufällige öffnet ${ }^{25}$ doch besteht die eigentliche Dignität des Kosmos in der Unveränderlichkeit seiner Strukturen, welche ihn wiederum zu einem möglichen Gegenstand

23 Tim. 59 c; cf. 29 b; 48 d; 53 d; 56 b; 57 d; 59 d; 72 d.

24 Etwa: 268 b 3 sq.; 269 a 19 sqq.; 272 a 22 sq.; 276 a 18 sqq.; etc.

${ }^{25}$ Buridan gesteht dies ausdrücklich zu: In der wichtigen Frage, ob omne futurum de necessitate eveniet, sagt Buridan, nachdem der eine Reihe von Pro-Argumenten aufgeführt hat: "oppositum tenet Aristoteles et fides catbolica“: Met. VI, 5 (35vb); cf. Periherm. I, 11 (cd. Van der Lecq p. 52). Buridan hat sogar - wie er selbst zugesteht - große Schwierigkeiten, den Nezessitarismus, den er mit Platon in Verbindung bringt, auszuräumen: 36ra; 36va. 
des Wissens macht. Jene Strukturen könnten aber nur dann als strikt unveränderlich gedacht werden, wenn konstitutive Elemente des christlichen Verständnisses der Schöpfung uminterpretiert würden. Gerade die Verurteilung von 1277 hat jedoch dafür gesorgt, die wissenschaftspsychologisch nachvollziehbare Attraktivität des von Gilson so genannten Nezessitarismus durch das Zutagetretenlassen seiner Bedingungen zu relativieren.

Buridans Naturphilosophie ist nun eines der prägnantesten Beispiele dafür, wie dieser Situation entsprochen werden kann. Es wäre jedoch ganz falsch, hierin lediglich die Konfrontation zweier durch geschichtliche Herkunft und geistige Verfaßtheit völlig disparater Mächte zu sehen. Denn die christliche Theologie der Hochscholastik nimmt einerseits den Grundbegriff der Möglichkeit aus dem aristotelischen Denken auf und transformiert ihn doch zugleich tiefgreifend. Denn wenn die Verfaßtheit und sogar die Existenz dieser Welt deswegen als nicht notwendig gedacht wird, weil der Ursprung von Existenz wie Verfaßtheit dieser Welt durch sein Wesen dazu nicht genötigt ist, dann ist diese Welt auch als wirkliche nur eine mögliche. Hier muß man sich den Sinn dieser Möglichkeit deutlich vor Augen führen. Die Möglichkeit dieser Welt besteht zuletzt nicht darin, $\mathrm{daß}$ sich zu ihr logisch äquivalente Alternativen denken lassen, sondern darin, daß durch ihren Ursprung eine andere Welt möglich wird, weil dieser sie hätte erschaffen können. Allerdings sind diese beiden Sinne von Möglichkeit nicht als exklusive Alternativen zu verstehen; denn ohne diese innere Möglichkeit ließe sich nicht angeben, worauf sich das göttliche Können beziehen soll, aber ohne dieses Können, diese „Macht“ blieben die anderen Möglichkeiten ontologisch unausgewiesen. Die Theorie, welche beides verbinden sollte, war die Lehre vom possibile logicum. Dieses meint - obwohl es den weitesten Raum von Möglichkeit definiert keine autonome Möglichkeit, denn sie ist nicht reine Gedachtheit. Durch jene Identifizicrung ist sie vielmehr im Sinne eines realen Könnens zu denken, welches daher als causa zu fungieren imstande ist.

Dabei ist wichtig, klar zu sehen, wie sich der neue Möglichkeitsbegriff zur aristotelisch gefaßten dynamis verhält. Buridans Auslegung zeigt nämlich, daß die Formel „potentia simpliciter" uneingeschränkt ernst zu nehmen ist. Die absolute Möglichkeit ist nicht eine „ganz andere“, für uns gänzlich unnachvollziehbare, vielmehr ist ihr Umkreis genau durch das bestimmt, was für uns die oberste Bendigung der Denkbarkeit ist: Gott kann wirklich das zum Sein bestimmen, was wir denken können. Diese Möglichkeit des göttlichen Handelns berücksichtigt Buridan in der Weise, daß erst dadurch der Status des Natürlichen gegen das Supernaturale abgegrenzt wird. Diese Kategorie muß jedoch für den Philosophen Buridan leer bleiben, weil ein nicht-natürliches Geschehen in seinem Wie für uns nicht aufklärbar ist. Allerdings ergibt sich aus seiner prinzipiellen Möglichkeit, daß auch die wirkliche Natur (gegen Aristoteles) nur kontingent verfaßt ist. 
Durch die Verbindung von Natur- und Notwendigkeitsbegriff war es daher unumgänglich geworden, die Naturphilosophie des Aristoteles eincr grundsätzlichen Revision zu unterziehen. Damit sind also nicht jene inhaltlichen Revisionen gemeint, die Buridan an vielen einzelnen Lehren anbringt und von denen seine Impetustheorie nur die prominenteste ist - wenngleich man erwarten kann, daß solche Korrekturen auf prinzipielleren Gedanken oder Einstellungen beruhen. Für unser Thema muß folgendes beachtet werden: Ein Lehrer der Artes-Fakultät wie Buridan setzt nicht die theologisch motivierte Spekulation um das possibile logicum fort. Einer der führenden Pariser Nominalisten vermag aber auch nicht die aristotelische Lehre von der Möglichkeit ohne Eingriff zu übernehmen. Es kann hier nicht auf den Begriff der Bewegung ausführlich eingegangen werden. $\mathrm{Daß}$ er nicht mehr der aristotelische sein kann, ergibt sich schon aus der Eliminierung des Potenzmomentes aus dem Seinsbegriff, welches für Aristoteles, wie eingangs gesagt, konstitutiv war. A. Maier hat die Interpretationsgeschichte der aristotelischen Bewegungsdefinition nachgezeichnet. ${ }^{26}$

Auf diese Weise wird zum einen der Naturphilosophie ein gewisses Eigenrecht eingeräumt. Es beruht auf einer programmatischen Ausklammerung theologisch relevanter Fragestellungen. Mit dieser Selbstbescheidung vermag sich also die Philosophie auch von sich aus eine gewisse Selbständigkeit zu sichern, zu deren Stabilität allerdings am Ende noch eine Bemerkung zu machen sein wird.

E. Grant, der bedeutende amerikanische Kenner der mittelalterlichen Wissenschaftsgeschichte, hat das Separationsmodell Buridans ganz auf institutionelle Bedingungen zurückführen wollen. Ein am 1. 4. 1272 erlassenes Statut der Pariser Universität hat den Magistern der Artes untersagt, theologische Fragen zu disputieren oder gar zu determinieren. ${ }^{27}$ Nun erwähnt Buridan sogar einmal diesen Eid, den alle Magistri zu Beginn ihrer Tätigkeit zu leisten hatten, als er von einem Einwand gegen seine Lehrweise berichtet. Er verteidigt sich aber damit, daß bei der Frage der Möglichkeit eines Vakuums nicht in vollständiger Weise alle theologischen Aspekte ohne Beeinträchtigung für die Erörterung ausgeklammert werden können. ${ }^{28}$ Wie aber auch die Vorgeschichte des Separationskonzeptes zeigt, kommt dem Interesse der Theologie an einer Selbstbeschränkung der Philosophie deren eigenes entgegen, ihre Betrachtung des natürlicherweise Seienden und Geschehenden nicht durch Einbezug übernatürlicher, und

${ }^{26}$ Motus est actus entis in potentia..., in: Zwischen Philosophie und Mechanik, Rom 1958, $3-57$.

${ }^{27}$ CUP nr. 441 (Denifle-Châtelain I p. 499).

${ }^{28}$ Phys. IV, $8(73 v-74 r)$; dazu E. Grant, The Condemnation of 1277, God's absolute Power, and Physical Thought in the late Middle Ages, in: Studies in Medieval Science and Natural Philosophy, London 1981, cap. XIII p. 232-235. 
insofern völlig kontingenter Möglichkeiten zu zerstören. Dies wäre einfach mit dem philosophischen Wissensanspruch nicht zu vereinbaren. Zudem könnte eine historisch breiter angelegte Untersuchung wohl unschwer plausibel machen, daß es nicht bloß eine Form, das Eigenrecht philosophischer Betrachtungsweise zu reklamieren und in Anspruch zu nehmen, gibt. Daher kann dieser Eid nicht als hinreichende Erklärung dienen. ${ }^{29}$ Gewiß könnte man auch im Zusammenhang der buridanischen Unterscheidung im Begriff der Möglichkeit auf den Syllabus von 1277 verweisen, dessen Satz 184 lautete: „quod possibile vel impossibile simpliciter, id est, omnibus modis, est possibile vel impossibile secundum philosopbiam." ${ }^{30}$ Doch würde man damit ipso facto die Plausibilität unterschätzen, die in einem Begriff der Möglichkeit als logischer Kohärenz liegt und welche nicht aufgeht in den Möglichkeiten, welche durch die schon bestehende Wirklichkeit eröffnet werden. Zumal jene Möglichkeit durch ein logisch äquivalentes Kriterium definiert wird, das - einmal formuliert - dem philosophischen Denken unmittelbar zugänglich ist, scheint es ohne weiteres nachvollziehbar, daß auch für Buridan in der jetzt vorfindlichen Wirklichkeit nicht alle Möglichkeiten erschöpft sind. ${ }^{31}$

Trotz des Votums für die theoretische Bedeutsamkeit als solcher seien einige Beispiele dafür gegeben, was Buridan durch die Allmacht möglich schien. Dies kann immer einen zweifachen Akzent haben. Entweder in einem separarierend-reduktiven Sinne (nu r übernatürlicherweise möglich) oder in einem eröffnenden Sinne (übernatürlicherweise immerhin möglich): Der Himmel ist in übernatürlicher Weise geschaffen und ebenso vernichtbar, aber, da er keine materia enthält, ist dies natürlicherweise nicht möglich. ${ }^{32}$ Darin liegt keine zur Konfliktvermeidung entworfene Zweigleisigkeit, die gewissermaßen unter der Generalklausel der Übernatürlichkeit die Wahrheit des christlichen Schöpfungsglaubens konzedieren, die aristotelische Kosmologie jedoch intakt lassen kann. ${ }^{33}$ Buridan erwähnt in den Erwiderungen ausdrücklich, daß die Zerstörbarkeit weniger ein außerhalb des aristotelischen Horizontes liegender Gedanke, sondern kraft seiner Prinzipien ein Gegenstand möglicher Bestreitung. Buridan sagt dazu: „quando dicitur quod deus potest velle non conservare caelum, ego concedo; ideo caelum est supernaturaliter annibilabile. Sed Aristoteles negasset quod Deus posset velle corrumpere vel annibilare caelum, propter boc quod est immutabilis

${ }^{29}$ McLaughlin hat die Tragweite dieser Eidesleistungen aufgrund von deren Vielzahl sehr relativiert: Intellectual Freedom and Its Limitations in the University of Paris in the Thirteenth and Fourteenth Centuries, New York 1977 (Phil. Diss. Columbia Univ. 1952), 23 sqq.

${ }^{30}$ Cf. R. Hissette, Enquête sur les 219 articles condamnés à Paris le 7 Mars 1277; LouvainParis 1977,275 sq.

31 Phys. I, 18 (22va): "ego suppono quod deus multas creaturas potest creare quas numquam creabit."

32 De caelo I, 10 (ed. Moody p. 46).

33 In diesem Sinne: De caelo I, 16 (Moody 77); I, 23 (Moody 116). 
voluntatis. Nec istud est contra libertatem suae voluntatis, quia Aristoteles reputat liberum simpliciter non quia potest inopposita, sed quia est sui ipsius gratia tanquam finis aliorum." ${ }^{34}$ Gott kann etwa auch mehrere Engel innerhalb derselben Species erschaffen. ${ }^{35}$ Gerade im Kommentar zu De caelo häufen sich die Unterscheidungen von dem, was natürlicherweise unmöglich ist, übernatürlich aber sein kann: daß ein im Kreis bewegter Körper unendlich sein kann; ${ }^{36} \mathrm{da}$ von ihm die ganze Welt in gerader Richtung bewegt werden könnte; ${ }^{37}$ daß Gott auch andere Welten neben dieser hätte erschaffen können; ${ }^{38}$ ob irgendetwas außerhalb des Himmels sein könnte, wird in die Kompetenz der theologischen Fakultät verwiesen; ${ }^{39}$ daß es mehrere Himmelsbewegungen geben könnte; ${ }^{40}$ es wird die Frage untersucht: was folgt für einen ein Fuß großen Körper und darüber hinaus für das Verhältnis von Substanz und Quantität, wenn Gott alles andere vernichten, diesen aber zu einem zwei Fuß großen Körper verdünnen würde? ${ }^{41}$ Für die göttliche Allmacht gibt es keinen höchsten Wärmegrad. ${ }^{42}$

Für den Sinn des Möglichkeitsgedankens bei Buridar: ist also, wie zu zeigen versucht wurde, von besonderer Wichtigkeit, daß seine Funktion ausschließlich und ausdrücklich auf die Statusbestimmung der Naturphilosophie beschränkt bleibt. Die Konzession des Nicht- und Übernatürlichen ist eine abstrakte, die nicht in den faktischen Vollzug der naturphilosophischen Erörterung eingeht. In einen größeren Kontext gebracht: Gehörte es zu den herausragenden Aspekten der Aristoteles-Kritik bei Duns Scotus, daß die reale Verfaßtheit des Menschen zwar zutreffend bestimmt, aber aufgrund der unvermeidlichen Unkenntnis der Erbsündenlehre in ihrem Status als bloß geschichtlicher und eben nicht „natürlicher" Bestimmtheit ebenso unvermeidlich überschätzt wurde, so ist auch die Natur selbst nicht von jener alternativlosen Determiniertheit wie es scheinen könnte. Dies ist kein Rückzug in eine bloß konstatierende Naturphilosophie. Es bedarf im Prinzip immer noch des Nachdenkens über Gründe. Diese sind jetzt nur von anderer Art. Es sind nicht die Gründe dafür, warum etwas so sein muß wie es ist, sondern dafür, warum es so ist wie es ist, obgleich es anders sein könnte. Daß diese nicht immer angebbar

34 De caelo I, 10 ad 2 (Moody 48).

35 De caelo I, 11 ad 4 (Moody 53).

36. De caelo I, 15 (Moody 70).

37. De caelo I, 16 (Moody 75): „sed de potentia divina determinatum fuit per episcopum Parisensis et per studium Parisiense, quod error esset dicere quod deus non posset movere totum mundum simul motu recto."

38 De caelo I, 18 (Moody 84).

39) De caelo I, 20 (ed. Moody p. 93): „sed quid sit dicendum de hoc secundum fidei veritatem sive constantiam, debetis recurrere ad theologos"; ähnliche Exklusion: De caelo I, 25 (Moody 123).

4) De caelo II, 10 (Moody 170).

41 Phys. I, 8 (11va).

42 Phys. I, 12 (15vb). 
sind, ist demgegenüber eher sekundär. Es kommt jedoch als zusätzliche Komponente der Rationalität die Reflexion auf den Status hinzu.

Allerdings kann man sich fragen, ob die Beschränkung auf das natürlicherweise Seiende tatsächlich bloß eine Statusbestimmung der Methode voraussetzt, oder ob sich schon daraus - und nicht erst aus der ohnehin inhaltlich orientierten Diskussion mit Zeitgenossen - eine Verengung der Naturphilosophie ergibt. Ist Buridans Naturphilosophie noch Kosmologie? Oder wird diese an die Schöpfungstheologie delegiert? Wenn Buridan die Schöpfung aus der Gültigkeit jener alten - und überall konzedierten Formel des Kausalprinzips ,ex nibilo nibil fit" ausnimmt, dann nicht, um - wie Thomas ${ }^{43}$ und viele andere - eine eigene Verursachung des Seiendseins zu statuieren, welche Gegenstand der Metaphysik wäre, sondern vielmehr, um sie schlechthin zum Gegenstand des Glaubens werden zu lassen. ${ }^{44}$ Zwar behält Buridan bei der Kausalität das kooperative Element des Zusammenwirkens von causa particularis und universalis bei, doch läßt sich letztere nicht als causa essendi fassen - selbst wenn es manchmal so aussieht. ${ }^{45}$ So bleibt es zunächst bei der korrekten Fixierung der Unterscheidungen: Schöpfung setzt nur den Schöpfer selbst voraus, während ein natürliches Hervorbringen (factio) nicht allein ein agens, sondern auch ein subiectum voraussetzt, „in dem es geschieht. “46 Doch geht Buridan darüber nicht mehr hinaus. Er weist ein Argument zurück, mit dem Thomas von Aquin im Rahmen einer metaphysischen Ontologie versucht

43 In Phys. VIII, 2 (974): „quia omnis motus indiget subiecto, ut bic Aristoteles probat et rei veritas babet, sequitur quod productio universalis entis a Deo non sit motus nec mutatio, sed sit quaedam simplex emanatio. Et sic fieri et facere aequivoce dicuntur in hac universali rerum productione, et in aliis productionibus. Sicut ergo si intelligamus rerum productionem esse a Deo ab aeterno, sicut Aristoteles posuit, et plures Platonicorum, non est necessarium, immo impossibile, quod buic productioni universali aliquod subiectum non productum praeintelligatur." Nicht bloß die Kompatibilität von Kausalsatz und Schöpfungslehre behauptet Thomas, sondern auch, daß bereits Platon wie Aristoteles die Grundstruktur kreativen Hervorgehens als Kausierung des Seins schlechthin - und bloß eines je bestimmten Seienden - eingesehen haben, demgegenüber der Zeitlichkeitscharakter ganz sekundär wird: nr. 975; VIII, 3 (996): "quamvis Aristoteles poneret mundum aeternum, non tamen credidit quod Deus non sit causa essendi ipsi mundo, sed causa motus tantum, ut quidam dixerunt."

44 Phys. I, 15 (18vb): „Et tunc ego pono duas conclusiones. Prima est quod possibile est aliquid fieri sine subiecto praesupposito ex quo vel in quo fiat et banc conclusionem credo fide et non aliqua probatione nisi hoc auctoritate sacrae scripturae et doctorum fidei catbolicae. Sic enim Deus fecit et creavit angelum et caelum et mundum et hanc conclusionem intendunt qui dicunt posse aliquid fieri ex nibilo vel etiam ex nibilo posse aliquid fieri. Secunda conclusio est quod necesse est omne quod fit naturaliter fieri ex subiecto praesupposito... sed tamen non puto quod baec conclusio sit demonstrabilis, sed est declarabilis per inductionem in qua non inventa est instantia; sic probat enim eam Aristoteles et tale reputari debet principium in scientia naturali."

${ }^{45}$ Met. I, 4 (5va); IV, 3 (14va - b); Eth. IX, 7 (200ra). - Buridan rekurriert auf eine geläufige Unterscheidung: Thomas von Aquin, Sent. I d. 7, 1, 1 ad 3; Heinrich von Gent, Sum. 24, 6 (141rN); 22, 5 (135rEF); Duns Scotus, ord. II d. 1 q. 3 (VII p. 57).

46 Phys. I, 21 (24vb). 
hatte, den Gedanken der Schöpfung philosophisch unumgänglich zu machen. Thomas hatte von einer unendlichen Distanz von Sein und Nichtsein gesprochen. Eine endliche Ursache ist jedoch gerade dadurch in ihrer Endlichkeit gekennzeichnet, daß sie zur Entfaltung ihres Wirkvermögens bereits auf Bestehendes angewiesen ist. ${ }^{47}$ Dieses Argument, das allerdings auch für Scotus schon nicht einleuchtend war, weist Buridan ohne Namensnennung als absurd zurück: ,absurdum est dicere quod sit infinita vel distantia aliqua de non ente ad ens; si enim sit aliqua distantia, ipsa est ens vel entia vel inter entia." ${ }^{48}$ Zugleich wendet sich Buridan aber auch gegen Averroes, der mit großer Mühe versucht hatte (laboravit), die Schöpfungslehre als innerlich unmöglich zu erweisen. Dessen Argumente werden als zirkulär zurückgewiesen, jedoch ohne daß ihnen stärkere gegenübergestellt würden. Dies geschieht aus grundsätzlichen Gründen: Was immer zum Gegenstand der Naturbetrachtung soll werden können, muß sich auf die Elemente der Induktion zurückführen lassen. Für Aussagen über den Kosmos als ganzem fehlt also jede Grundlage, durch die gesagt werden könnte, ob sie wahr oder falsch sind. Der andere oben angedeutete Weg einer metaphysischen Konzeption von Ursächlichkeit ist nur für die historische Perspektive eine Alternative, für Buridan wird dies unter der Voraussetzung eines bestimmten Verständnisses von ens (dem wir hier nicht nachgehen können) gar nicht zum Gegenstand der Erwägung. Daher bleibt konsequenterweise ausschließlich die Möglichkeit, die kreative Form der Statuierung als miraculose zugleich zuzugestehen wie auch aus der theoretischen Durchdringung zu eliminieren. Die Naturphilosophie im Konzept Buridans muß auf den Gedanken einer Schöpfung verzichten. Sie kann ihn mit ihren Mitteln weder statuieren noch kritisieren.

Die Fundamentalaufgabe der Metaphysik, nämlich die Gewinnung eincs angemessenen Wirklichkeitsbegriffs, macht diese unter der Voraussetzung einer bedingten Autonomie selbst zweideutig. Denn die Konzeption eincs zwar autarken Wissens, welches zu den Gehalten des Glaubens kein Wissens-, sondern nur ein Anerkennungsverhältnis zu gewinnen imstancle ist, muß hierdurch stark relativiert werden. Denn es ließe sich etwa am Beispiel von Buridans Diskussion der Ontologie des Akzidens ${ }^{49}$ leicht zeigen, daß das Selbstverständnis der Metaphysik als Form, und zwar als Höchstform des Wissens, nicht dadurch zu stabilisieren ist, daß durch eine bewußte, d. h. methodische Abstraktion von theologischen Bestimmungen, von diesen freigehalten wird. Dieses Modell kollidiert am Ende also doch mit den theoretischen Ansprüchen, die von der Metaphysik einerseits

4. Sth. I, 45,5 ad 3.

48 Phys. I, $15(19 \mathrm{rb})$.

49 Met. IV, 6: "utrum boc nomen ens significet substantias et accidentia secundum unam rationem sire" secundum unum conceptum." 
und der Theologie andererseits erhoben werden. Hatte Duns Scotus versucht, solche Kollisionen zu vermeiden, indem er einerseits die philosophische Erkenntnismöglichkeit beschränkte, andererseits eine weitgehend entkosmologisierte Metaphysik zu entwickeln versuchte, so zeigt sich jetzt, $\mathrm{d} a ß$ auch dies noch nicht hinreichend war.

Das Denken verzichtet auf eine Einheitsleistung, von der man weiterhin unterstellt, sie müsse möglich sein und daher auch durchgeführt werden. Die Explikation dieser nicht-natürlichen, sondern mirakulösen Wirklichkeit, wird bei Buridan nicht geleistet. A. Ghisalberti hat dieses Buridan zum Vorwurf gemacht. ${ }^{50}$ Wenn aber die hier vorgetragene Interpretation Buridans angemessen ist, dann geht dieser Vorwurf an Buridans Intention vorbei. Gewiß, eine den sog. „Synthesen“ des 13. Jahrhunderts vergleichbare Zuordnung bleibt aus. Diese jedoch stammten allesamt von Theologen, und ein solcher ist Buridan nicht. Immerhin gehört Buridan einer sehr formalen Strukturbestimmung nach noch ganz in diese Tradition. Es ist eine noch kaum wahrgenommene Gemeinsamkeit der großen Autoren der Hochscholastik, daß sie die Wahrheit des Glaubens nicht als eine gegenüber der endlichen Rationalität ganz andere angesetzt haben. Jene ist zwar durch diese nicht erreichbar, doch qua Wahrheit liegt sie „über der Vernunft." ${ }^{51}$ Dies gilt auch für den Philosophen Buridan.

Die Philosophie Buridans stellt nur die Kategorie des Non-Naturalen bereit; sie vermag aber nicht diejenigen Fälle positiv zu explizieren, in denen auf diese Kategorie zurückgegriffen werden muß. Diese Explikation kann für Buridan nur eine theologische sein. Mehr als die Konzession der Möglichkeit, nämlich eine inhaltliche Präjudizierung, wäre nicht mehr das, was Buridans Denken zu sein beansprucht, nämlich Philosophie. Sie ist allerdings eine Philosophie, die davon ausgeht, daß die Vernunft über die Generalhypothese der Allmacht Gottes verfügt. Buridan revidiert nicht die seit Scotus als zugestanden geltende Unbeweisbarkeit der göttlichen Omnipotenz. Fs bedarf weder einer Plausibilisierungsleistung noch sonst ciner Weise vernünftiger, d. h. legitimierender und nicht bloß faktischer Integration. Daß Gott allmächtig ist, ist für Buridan ohne Einschränkung die Wahrheit. Eine solche weiter nicht integrationsfähige, aber anerkannte Wahrheit wird zu so etwas wie einem Faktum. Es muß einfach als ein Gegebenes berücksichtigt werden. Die Form dieser Berücksichtigung ist dann aber die bereits aus anderen Autoren bekannte: Sie wird als ein logisches Äquivalent zur Widerspruchsfreiheit genutzt. Weil dem aber so

50) La teologia di Giovanni Buridano, in: Riv. di filos. neoscol. 65 (1973), 17-53, p. 47 n. 58.

5t Supra rationem: Bonaventura, in Hexaëm. XIX, 14 (V, 422 b); Albertus Magnus, s. Eth. I, 13 n. 80 (ed. Col. XIV, 1 p. 71); Thomas von Aquin, Sent. IV d. 10, expos. textus; s. Matth. XVI, 2 (1379); Siger v. Brabant, de an. int. 7 (ed. Bazán p. 108); in Met. III, 5 (Rep. Par.) (ed. Maurer p. 412). 
ist, wird das schlechthin Mögliche nicht zu einem völlig vernunftexternen Gesichtspunkt. Erst wenn das supernaturale kriteriell nicht mehr einzuschränken wäre, dürfte von einem voluntaristischen Theologismus die Rede sein. H. Blumenberg hat nun tatsächlich und ohne Einschränkung Buridan dieser Richtung zugerechnet. Der Text allerdings, den er dafür als Beleg anführt - „cum nibil scias de voluntate dei tu non potes esse certus de aliquo" - , findet sich zwar in Buridans Metaphysik-Kommentar, aber unter denjenigen Argumenten, die seiner eigenen These gerade entgegenstehen. ${ }^{52}$

Das philosophische Wissen kann zum Glauben nur ein faktisches Verhältnis einnehmen. Der Glaube ist für Buridan eine Wahrheitsform, zu der sich das Wissen nicht in ein Verhältnis bringen kann, das wiederum von der Art des Wissens sein könnte. Die Wahrheit kann nur anerkannt werden. Darin ist also die Autarkie des philosophischen Wissens beschränkt. Sic ist aber keine, die sich dieser Anerkennung erst verdankte. Sie ist also zwar beschränkt, aber nicht dependent. Philosophie ist zum einen die höchste Form des Wissens, das Wissen aber nicht die einzige Form der Wahrheit. Diese Autarkie macht sich, wie gesagt, gerade darin geltend, daß die Grenzen philosophischer Kompetenz strikt eingehalten werden.

$\mathrm{Daß}$ kraft Gottes Allmacht alles logisch Mögliche auch real möglich werden könnte, kann die Philosophie im Verständnis Buridans nicht erreichen. Wenn sie sich aber aus dem Glauben eben dies vorgeben läßt, daß sich Gott wirklich auf alles das beziehen kann, das keinen logischen Widerspruch einschließt, dann vermag sie den ontologischen Status des natürlichen Geschehens zutreffend zu bestimmen. Dieser Gesichtspunkt des Möglichen hatte schon in der hochscholastischen Erörterung des Verhältnisses von Glaube und Vernunft eine wesentliche Rolle gespielt, wenn auch gleichsam in umgekehrter Richtung. Sowohl Thomas von Aquin wie Duns Scotus ordnen der Vernunft die Funktion zu, zwar nicht die Einsicht in die Gründe des faktisch zu Glaubenden, wohl aber dic Möglichkeit des Glaubensinhaltes darzutun. ${ }^{53}$ Die Dominanz des Mög. lichkeitsdenkens, die sich bereits aus der Reformation der Metaphysik bei Duns Scotus ergeben hat, erhält somit aus Buridans Konzept der Naturphilosophie zusätzliche Schubkraft.

Im ganzen betrachtet scheint Buridans Konzept seiner Philosophie doch cin instabiles zu sein. Die Verselbständigung der Philosophie im Mittelaltcr, welche bei Buridan innerhalb der Scholastik auf einem respektablen Niveau $\mathrm{zu}$ studieren ist, wird unter sehr massiven Konditionen versucht. Wenn sie zutreffen sollte, dann ließe sich aber auch ein anderes Faktum der

52 Die Genesis der kopernikanischen Welt, Frankfurt 1965, p. 184; cf. Met. II, 1 (8va).

53 Thomas, in de trin. 2, 1 ad 5; sth. II-II, 2, 10 ad 2: ,rationes quae inducuntur ad auctoritatem fidei, non sunt demonstrationes, ... sed removent impedimenta fidei, ostendendo non esse impossibile quod in fidei proponitur"; Duns Scotus, ord. II d. 1 n. 139 (VII p. 70). 
Philosophiegeschichte verständlich machen. Eines der Charakteristika der frühneuzeitlichen Denkgeschichte ist das umfängliche Aufgreifen theologischer Themen in der Philosophie. Dafür stehen nicht allein Descartes und Leibniz. Dies ließe sich aus der Perspektive des 14. Jahrhunderts als der Versuch verstehen, die durch Ausgrenzung zwar erreichte, aber bloß fragile Eigenständigkeit der Philosophie durch Integration von Theologumena zu einer stabilen zu machen. 